\title{
Systemic sarcoidosis with refractory ventricular tachycardia and heart failure
}

Sir,

We read with interest the recent case report of Walsh (British Heart fournal, 1978, 40, 931-933). We have recently seen 2 patients with sarcoidosis in whom similar clinical evidence strongly suggested a diagnosis of myocardial sarcoidosis. However, more extensive investigations failed to support this in either case.

The first patient is reported elsewhere (Johnson et al., 1978). He was a West Indian who presented with bilateral pleural effusions. Histological evidence for sarcoidosis was obtained from pleural, liver, and mediastinal lymph node biopsies. The Kveim test was positive. When he was first seen, there was a fourth heart sound but no other cardiovascular abnormalities. Six weeks later he developed a harsh apical early systolic murmur characteristic of mitral regurgitation. We strongly suspected this to be caused by myocardial sarcoidosis. Further investigations including echocardiography, cardiac catheterisation, and coronary arteriography showed haemodynamically insignificant mitral regurgitation. Numerous endomyocardial biopsies were taken from the left ventricle, but did not show sarcoidosis either on light or electron microscopy. The patient has returned to normal health with steroid therapy but his cardiac murmur persists.

Our second patient was diagnosed as having sarcoidosis in 1969 after having a routine mass $x$-ray film which showed bilateral hilar lymphadenopathy. The diagnosis was confirmed by mediastinal lymph node biopsy and positive Kveim test. At no time have there been any abnormal physical findings apart from pulse irregularities. Her electrocardiogram in 1969 showed ventricular and atrial ectopic beats and widespread non-specific $T$ wave changes. On this evidence a diagnosis of myocardial sarcoidosis was made; she has been treated intermittently with steroids over the past 9 years, during which time palpitation and electrocardiographic abnormalities have been variably present. In view of the negative findings in our first patient, we decided to investigate this patient further. A sector scan and $M$ mode echocardiographic examination of her heart showed the classical features of anterior and posterior mitral leaflet prolapse; there was also a small area of apical asynergy. During a one-hour electrocardiographic recording she had many multifocal ventricular premature beats. In view of these findings, we intend to discontinue steroid therapy and to treat her with beta-blockers.

Although we cannot be absolutely certain that neither of our patients has localised myocardial sarcoid infiltration, we feel that our extensive investigations make it unlikely and we now regard these cardiac abnormalities as coincidental with sarcoidosis. In view of the similarities between our cases and that of Walsh (1978), we think that more extensive investigations such as echocardiography or cardiac catheterisation with coronary angiography and cardiac biopsy should be undertaken before cardiac abnormalities in patients with sarcoidosis are attributed to myocardial involvement. This is particularly important, firstly because long-term steroid treatment may lead to a steroid cardiomyopathy, and secondly because there is some evidence to suggest that steroid treatment in myocardial sarcoidosis may increase the risks of development of ventricular aneurysm (Roberts et al., 1977).

N. McI. Johnson, M. W. McNicol,
and D. J. Coltart
Cardiothoracic Department,
Central Middlesex Hospital,
London, NW10; and
Cardiac Department,
St Thomas' Hospital, London SE1.

\section{References}

Johnson, N. McI., Martin, N. D. T., and McNicol, M. W. (1979). Sarcoidosis presenting with pleurisy and bilateral pleural effusions. In the press.

Roberts, W. C., McAllister, H. A., jun, and Fertans, V. J. (1977). Sarcoidosis of the heart. A clinico pathological study of 35 necropsy patients and review of 78 previously described necropsy patients. American fournal of Medicine, 63, 86-108

This letter was shown to Dr Walsh who replies as follows:

Sir,

In their letter Johnson et al. comment on the similarity of their patients to mine. The patient I reported on was remarkable for the variety of cardiac disturbances and arrhythmias, including recurrent refractory ventricular tachycardia and fibrillation, and the development of heart failure; the paroxysmal 
rather than the persistent nature of these abnormalities was commented on. Refractory ventricular tachycardia has previously been described in patients with cardiac sarcoidosis but is an uncommon phenomenon in reversible cardiac disease other than acute myocardial infarction or drug intoxications.

The first case of Johnson et al. had negative endomyocardial biopsies. Cardiac sarcoidosis may be lethal even when the lesions are localised, for example in the conduction pathways. In a patient with only mitral regurgitation as a clinical and angiographic finding, I would be very surprised if endomyocardial biopsy were positive; failure to find granulomas does not deny the validity of their clinical suspicions. The value of cardiac biopsy is in the diagnosis of diffuse disease of the heart.

In their second patient, they do not describe the extensive investigations carried out, but refer only to echocardiography. This investigation was unhelpful in the patient I reported.

For the clinician the situation in a patient such as I described is straightforward; when there are refractory life threatening rhythm disturbances and heart failure, one cannot withhold treatment which might be beneficial. I hope that the long term followup of such patients by Fleming and others may provide information concerning the management of less seriously affected patients.

Michael J. Walsh, Wadsworth Hospital Center, UCLA School of Medicine, Wilshire and Sawtelle Blvds, Los Angeles, California 90073, USA 